\title{
Evidências iniciais de validade da escala de liderança virtuosa
}

\author{
Initial validity evidence of the Virtuous \\ Leadership Scale
}

\author{
Márcio GUIMARÃES ${ }^{1}$ iD 0000-0001-5785-9332 \\ Maria Cristina FERREIRA ${ }^{1}$ (iD) 0000-0003-0752-6710 \\ Michelle PEREIRA' ${ }^{1}$ iD 0000-0003-2437-2071
}

\section{Resumo}

O estudo objetivou reunir evidências iniciais de validade no contexto brasileiro para a Escala de Liderança Virtuosa. Participaram do estudo 417 trabalhadores de ambos os sexos (63\% do sexo masculino), do estado do Rio de Janeiro. As idades variaram de 18 a 72 anos $(M=35,6 ; D P=10,43)$. A análise fatorial confirmatória evidenciou que a versão brasileira da escala apresentou validade de estrutura interna. A Escala foi composta por cinco fatores de primeira ordem (coragem, prudência, temperança, humanidade e justiça), distribuídos por 18 itens que reproduziram integralmente a estrutura original do instrumento. Todas as subescalas apresentaram correlações positivas com a liderança autêntica, a satisfação no trabalho e os afetos positivos, e negativas com os afetos negativos. Conclui-se que a Escala de Liderança Virtuosa apresentou boas qualidades psicométricas em uma amostra brasileira.

Palavras-chave: Ética; Liderança; Psicologia positiva; Virtudes.

\begin{abstract}
The aim of the study was to collect initial validity evidence of the Virtuous Leadership Scale in the Brazilian context. A total of 417 workers of both sexes (63\% male) from the State of Rio de Janeiro participated in the study. The ages ranged from 18 to 72 years $(M=35.6, S D=10.43)$. Confirmatory factor analysis showed that the Brazilian version of the scale presented internal structure validity and it was composed of five first-order factors (courage, prudence, temperance,

\footnotetext{
$\checkmark \nabla v$

${ }^{1}$ Universidade Salgado de Oliveira, Departamento de Psicologia, Programa de Pós-Graduação em Psicologia. R. Marechal Deodoro, 217 , Bloco A, Centro, 24030-060, Niterói, RJ, Brasil. Correspondência para/Correspondence to: M. GUIMARÃES. E-mail: <epitafio9161@gmail.com>. Artigo elaborado a partir da dissertação de M. GUIMARÃES, intitulada "Liderança virtuosa e engajamento no trabalho em policiais militares". Universidade Salgado de Oliveira, 2016.

Como citar este artigo/How to cite this article

Guimarães, M., Ferreira, M. C., \& Pereira, M. (2019). Evidências iniciais de validade da escala de liderança virtuosa. Estudos de Psicologia (Campinas), 36, el70101. http://dx.doi.org/10.1590/1982-0275201936e170101
} 
humanity and justice). The 18 items replicate the original structure of the instrument. All subscales showed positive correlations with authentic leadership, job satisfaction and positive effects, and negative correlations with negative effects. It was concluded that the Virtuous Leadership Scale presented good psychometric qualities in the Brazilian sample.

Keywords: Ethics; Leadership; Positive psychology; Virtues.

Foi objetivo do presente trabalho reunir evidências de validade de estrutura interna, de relação com variáveis externas (American Educational Research Association, American Psychological Association, \& National Council on Measurement in Education, 2014) e de consistência interna da Escala de Liderança Virtuosa (Wang \& Hacket, 2016), em uma amostra brasileira. A pesquisa justifica-se pelo fato de que a consulta às bases de dados nacionais (SciELO e PePSIC), realizada em novembro de 2017, apontou a inexistência de estudos com essa finalidade, em que pese a importância da avaliação da liderança virtuosa no contexto organizacional.

O estudo da liderança virtuosa surge a partir da relevância conferida atualmente aos comportamentos éticos no âmbito dos negócios (Crossan, Mazutis, \& Seijts, 2013) e tem ensejado um crescente interesse no aprofundamento de investigações focalizadas na liderança com ênfase em virtudes. Tal estilo de liderança tem por base os preceitos de ética e justiça (Cameron, 2011) e vem sendo abordado há muito tempo pe los angeles antigos filósofos e pensadores religiosos que discorriam sobre sabedoria, coragem, humanidade, justiça, temperança e transcendência (Peterson \& Seligman, 2004).

A liderança virtuosa consiste, portanto, na adoção de medidas que garantam a justiça e a honestidade e que propiciem aos indivíduos e organizações o alcance de objetivos justos e morais (Cameron, 2011). Nesse sentido, os líderes virtuosos procuram tomar as decisões corretas, dentro dos prazos estabelecidos (Barclay, Markel, \& Yugo, 2012), razão pela qual costumam manter relações equilibradas com seus liderados (Prottas, 2013).

O líder virtuoso deve ainda demonstrar suas virtudes intencionalmente, de forma consistente e por razões intrínsecas (Whetstone, 2001). Nesse sentido, as ações devem ser intencionais, isto é, ele deve ter ciência dos fatos pertinentes a uma determinada situação. Além disso, o motivo da ação virtuosa deve ser intrínseco, ou seja, ela não pode ser adotada nem para proveito pessoal e nem tampouco como resultado de regras externas, controles ou compulsão. Por fim, a ação virtuosa deve ser expressa de forma consistente ao longo do tempo (Hart, 2001).

De acordo com Riggio, Zhu, Reina e Maroosis (2010), um líder virtuoso é aquele cujas características e ações são consistentes com quatro virtudes: prudência, fortaleza, temperança e justiça. A prudência está relacionada ao nível de precaução na tomada de atitudes, enquanto a fortaleza caracteriza-se pelo fato de um indivíduo suportar as intempéries do trabalho. A temperança, por sua vez, diz respeito à capacidade de lidar com os problemas mantendo o equilíbrio, e a justiça, por fim, consiste na adoção de decisões pautadas no que é estabelecido como mais correto.

Referenciando-se à teoria da aprendizagem social, em que os modelos são vistos como uma fonte indispensável de aprendizagem (Bandura, 1977) e Hackett e Wang (2012) propõem que as quatro virtudes cardinais descritas por Riggio et al. (2010) sejam revisadas, considerando as tradições confuncionista e aristotélica. Nesse sentido, os autores consideram que a liderança virtuosa compreende seis virtudes: coragem, humanidade, veracidade, prudência, justiça e temperança.

Nesse novo modelo teórico, a prudência, a temperança e a justiça mantêm o mesmo sentido a elas atribuídas por Riggio et al. (2010). A fortaleza, porém, é substituída pela coragem, caracterizada como um traço de caráter associado ao fato de o líder não ter medo de fazer o que acredita ser o correto, como, por exemplo, adotar ações impopulares ou que causem risco pessoal. Hackett e Wang (2012) acrescentam, ainda, 2 ao modelo original de Riggio et al. (2010), a humanidade, definida como um traço de caráter associado 
à capacidade de amar, de respeitar e de cuidar do outro, de modo desvinculado do próprio interesse; e a veracidade, conceituada como uma virtude inerente àquele que mantém as suas promessas e não engana as pessoas com as quais se relaciona, com o intuito de auferir vantagens.

Fundamentando-se no modelo teórico de seis virtudes, Wang e Hackett (2016) desenvolveram a Escala de Liderança Virtuosa, composta por 18 itens dispostos em seis fatores: coragem, humanidade, veracidade, prudência, justiça e temperança. Inicialmente, os autores realizaram uma análise comparativa das virtudes aristotélicas e confucionistas e buscaram suas equivalências, para propor uma lista com 89 itens, na qual pelo menos 14 comportamentos foram selecionados para descrever cada uma das seis virtudes do modelo.

Uma versão inicial do instrumento foi aplicada a 348 alunos de graduação na área de Recursos Humanos, os quais foram solicitados a responder aos itens tendo como referência um líder que tivessem observado durante qualquer experiência anterior de trabalho remunerado ou não. Os resultados da análise fatorial exploratória com rotação oblíqua revelaram a existência de cinco fatores, compostos pela aglutinação de 29 itens, na medida em que nenhum dos itens da dimensão veracidade saturou no fator apropriado.

Análises fatoriais confirmatórias realizadas em outro estudo, com a participação de 194 alunos de MBA de uma universidade norte-americana, levaram à eliminação de mais 11 itens. Desse modo, a versão final do instrumento, que apresentou bons índices de ajuste, ficou constituída por 18 itens, distribuídos entre cinco fatores correlacionados: coragem (quatro itens), prudência (quatro itens), temperança (quatro itens), justiça (três itens) e humanidade (três itens). Tais fatores apresentaram índices de consistência interna iguais a 0,$90 ; 0,88 ; 0,84 ; 0,86$ e 0,87 , respectivamente.

No processo de validação convergente, os escores de virtuosidade do líder, avaliados pelo subordinado, apresentaram correlações positivas altas com a autoavaliação dos líderes acerca de suas virtudes $(r=0,64$; $p<0,001)$. Por outro lado, correlações negativas moderadas foram obtidas entre a avaliação da virtuosidade do líder por seus subordinados e a autoavaliação dos líderes acerca de seu grau de maquiavelismo $(r=-0,37$; $p<0,001)$.

Considerando-se que o líder autêntico define-se por atributos de caráter e integridade que conferem direção aos subordinados e os ajudam a encontrar um significado para seus trabalhos e suas vidas (Sobral \& Gimba, 2012), hipotetizou-se que a liderança virtuosa apresentaria uma alta correlação positiva com a liderança autêntica (H1). Outrossim, o líder virtuoso, por suas características intrínsecas, costuma despertar reações emocionais e atitudes positivas em seus subordinados (Hacket $\&$ Wang, 2012), razão pela qual formularam-se as hipóteses de que haveria uma correlação positiva alta da liderança virtuosa com a satisfação no trabalho $(\mathrm{H} 2)$ e com os afetos positivos (H3), além de uma correlação negativa alta com os afetos negativos (H4). Tais hipóteses justificam-se pelo fato de que o líder virtuoso costuma adotar ações intencionais (Whetstone, 2001), que levam à manutenção de relações equilibradas com seus liderados (Prottas, 2013), o que tende a suscitar reações e atitudes positivas e a mitigar as reações e atitudes negativas de seus subordinados.

\section{Método}

\section{Participantes}

Para inclusão no estudo, utilizou-se o critério de o participante estar trabalhando há pelo menos um ano com seu chefe imediato em suas respectivas funções. A amostra foi composta por 417 trabalhadores brasileiros de diversas profissões e de ambos os sexos (63,0\% do sexo masculino), provenientes de diferentes regiões do Estado do Rio de Janeiro. A idade dos participantes variou de 18 a 72 anos $(M=35,6 ; D P=10,43)$

e sua maior escolaridade foi de nível médio $(47,0 \%)$, seguida do nível superior completo ou incompleto 
(21,0\%). No que diz respeito ao ramo da atividade, 53,1\% estavam ligados ao setor privado (indústria, comércio, prestação de serviços e financeiro), enquanto $71,5 \%$ estavam posicionados em cargos de nível administrativo ou operacional.

\section{Instrumentos}

A liderança virtuosa foi avaliada pela Escala de Liderança Virtuosa (LVS) de Wang e Hackett (2016), a ser respondida em escalas tipo Likert de cinco pontos, variando de "muito raramente" (1) a "muito frequentemente" (5). Os participantes foram solicitados a avaliar a frequência com que seu chefe imediato apresentava os comportamentos descritos em cada uma das afirmações da escala (Tabela 1). Exemplo de item: Meu chefe imediato demonstra preocupação e cuidado com seus pares. Na adaptação da escala para o português do Brasil, ela foi traduzida da língua inglesa para o português, por dois profissionais bilíngues da área da Psicologia, retraduzida para o inglês por outros dois profissionais bilíngues e, em seguida, submetida a um consenso final, mediante a análise de outro profissional da área de Psicologia (Borsa, Damásio, \& Bandeira, 2012).

Para medir a liderança autêntica foi utilizada a Escala de Liderança Autêntica (ALQ, Authentic Leadership Questionnaire) de Walumbwa, Avolio, Gardner, Wernsing e Peterson (2008), adaptada para amostras brasileiras por Cervo, Mónico, Santos, Hutz e Pais (2016). O instrumento compõe-se de 16 questões, destinadas a avaliar as quatro dimensões de liderança autêntica: autoconsciência; transparência; moral e ética; processamento balanceado. Tais itens são respondidos em escalas tipo Likert de cinco pontos, variando de "nunca" até "frequentemente". Exemplo de item: Meu chefe imediato admite erros quando eles ocorrem. No estudo original de construção, a escala apresentou índices de consistência interna iguais a: 0,73 (autoconsciência); 0,77 (transparência relacional); 0,73 (moral e ética); 0,70 (processamento balanceado). No presente estudo, os índices de consistência interna dos fatores foram iguais a 0,84 (autoconsciência); 0,82 (transparência); 0,84 (moral e ética) e 0,86 (processamento balanceado).

A satisfação no trabalho foi avaliada pela Escala de Satisfação Geral no Trabalho (Silva \& Ferreira, 2009), composta de cinco itens, a serem respondidos em escalas do tipo Likert de sete pontos, variando de "concordo fortemente" (1) a "discordo fortemente" (5). Exemplo de item: Eu recomendaria meu trabalho atual a qualquer pessoa que me perguntasse sobre ele. A escala apresentou consistência interna de 0,80 no estudo original, e de 0,84, no presente estudo.

Os afetos positivos e negativos foram avaliados pela Escala de Afetos Positivos e Negativos de Daniels (2000), adaptada ao contexto brasileiro por Ferreira, Silva, Fernandes e Almeida (2008). Ela se compõe de vinte itens, a serem respondidos em escalas tipo Likert de cinco pontos, variando de nunca (1) a sempre (5). Exemplos de itens: Inspirado, Furioso. Os itens são distribuídos em duas dimensões (afetos positivos e afetos negativos) que, na versão de Ferreira et al. (2008), apresentaram índices de consistência de 0,93 e 0,90, respectivamente. No atual estudo, esses índices foram iguais a 0,90 e 0,88, respectivamente.

\section{Procedimentos}

A coleta de dados foi totalmente realizada em versão online, com o questionário sendo inserido no aplicativo Google Docs e os convites à participação realizados por e-mail, facebook e whatsapp. A concordância prévia sobre a participação na pesquisa efetivou-se mediante o preenchimento do Termo de Consentimento Livre e Esclarecido.

Na análise de dados, foi realizada a Análise Fatorial Confirmatória, por meio da Modelagem de Equações

4 Estruturais, mediante o software MPLUS, versão 5.21 (Muthen \& Muthen, Los Angeles, CA, EUA), sendo 
testado o modelo constituído por cinco dimensões de primeira ordem e analisados os seguintes índices: Quiquadrado, Root-Mean-Square Error of Approximation (RMSEA), Comparative Fit Index (CFI) e Tucker-Lewis Index (TLI). Foram adotados, como bons índices de ajustes, valores abaixo de 0,08 para o RMSEA, e valores maiores que 0,95 para o CFI e para o TLI (Byrne, 2012). A consistência interna do instrumento foi analisada por meio do coeficiente Alfa de Cronbach.

As evidências de validade da escala por meio da relação com variáveis externas foram investigadas mediante o cálculo de correlações ( $r$ de Pearson) entre os escores do instrumento e os escores globais das medidas a ele relacionadas. Na avaliação da magnitude dessas correlações, foram adotados os critérios estabelecidos por Cohen (1988), que classifica as correlações em baixas $(0,10$ a 0,29), moderadas $(0,30$ a 0,49 ) e altas (acima de 0,50). Cumpre registrar que a pesquisa foi inicialmente submetida ao Comitê de Ética em Pesquisa da Universidade Salgado de Oliveira sob o protocolo de n 61998916.0.0000.5289, tendo sido aprovada.

\section{Resultados}

Tomando por base estudos de Wang e Hacket (2016), foram testados um modelo de cinco fatores de primeira ordem, um modelo de cinco fatores de primeira ordem com um fator de segunda ordem (considerando-se que os fatores poderiam se apresentar correlacionados e explicados por um fator mais geral) e um modelo unifatorial.

Tabela 1

Cargas Fatoriais Padronizadas dos Itens da Escala de Liderança Virtuosa

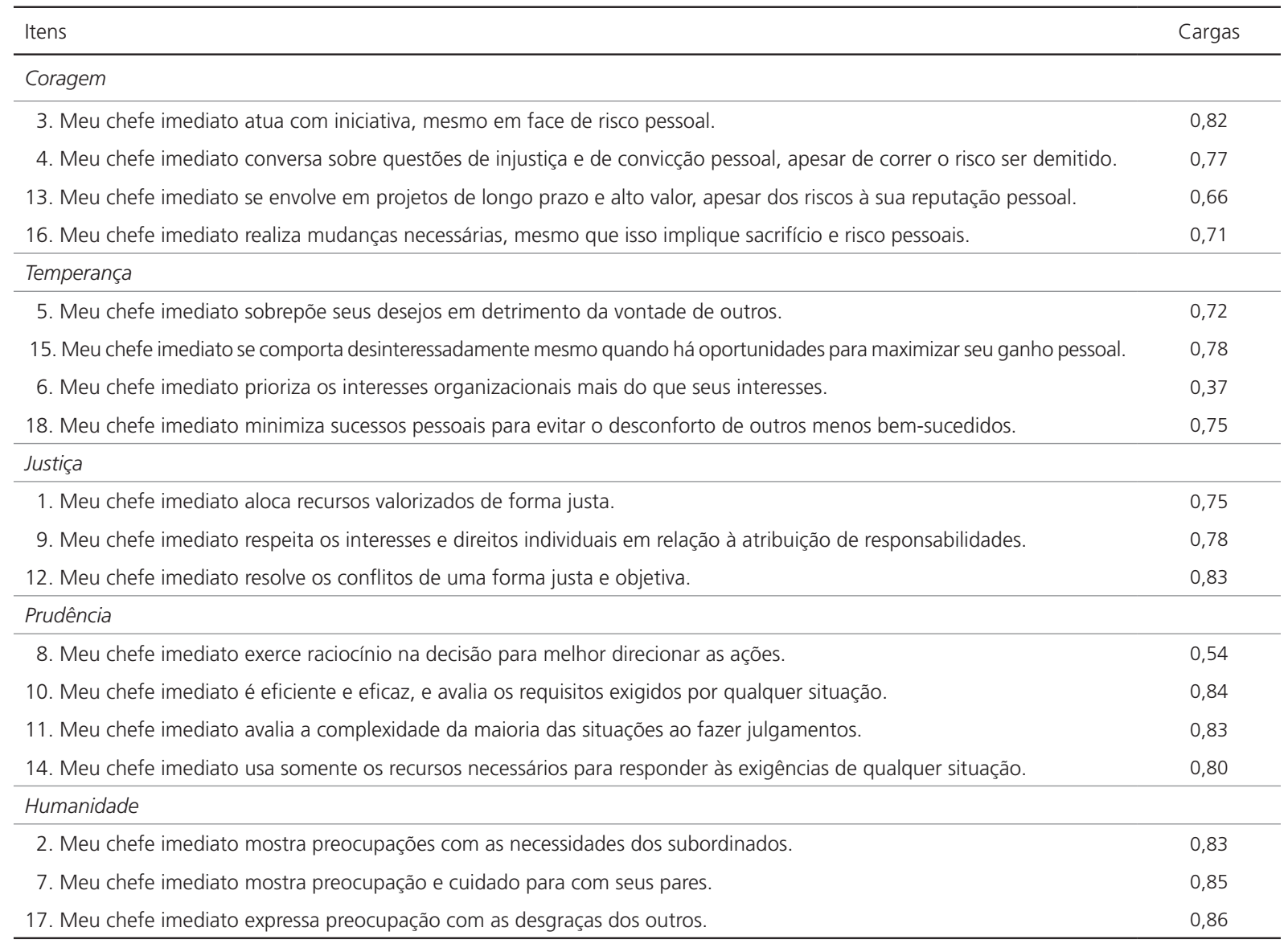


O modelo de cinco fatores de primeira ordem reproduziu integralmente a estrutura original do instrumento e apresentou índices de ajuste aceitáveis: $\chi^{2}=357,80(12)$; RMSEA =0,07; TLI =0,94; CFI =0,95 (Tabela 2), muito embora o TLI tenha ficado ligeiramente abaixo de 0,95. Além disso, os índices TLI e CFI do referido modelo foram ligeiramente superiores aos obtidos nos demais modelos testados, além de que as diferenças de qui-quadrado entre ele e os demais modelos foram significativas. Acrescente-se a isso o fato de que tal modelo é o que melhor reproduz a estrutura teórica proposta por Wang e Hacket (2016), para quem a liderança virtuosa manifesta-se por meio de cinco virtudes correlacionadas. Por essa razão, ele foi o modelo escolhido para representar a estrutura interna da escala.

A Tabela 1 apresenta as cargas fatoriais padronizadas de tal modelo, as quais foram todas significativas $(p<0,01)$ e, em sua maioria, variaram de 0,66 a 0,86, com somente dois itens ficando fora dessa faixa, mas, ainda assim, dentro de valores aceitáveis $(0,37$ e 0,54) (Brown, 2015). Os índices de consistência interna dos fatores, calculados pelo coeficiente Alfa de Cronbach, foram iguais a 0,81 (coragem), 0,75 (temperança), 0,82 (justiça), 0,83 (prudência) e 0,88 (humanidade).

Para a verificação da relação da escala de Liderança Virtuosa com variáveis externas, calcularam-se as correlações entre as diferentes escalas do estudo (Tabela 3). Conforme previsto, todos os fatores da escala de Liderança Virtuosa apresentaram correlações positivas altas com todos os fatores da Escala de Liderança

Tabela 2

Índices de Ajuste dos Diferentes Modelos Testados

\begin{tabular}{lcccc}
\hline Índices & $\chi^{2}(g l)$ & TLI & CFI & RMSEA \\
\hline Unifatorial & $519,45(135)$ & 0,91 & 0,92 & 0,08 \\
Primeira ordem & $357,80(125)$ & 0,94 & 0,95 & 0,07 \\
Segunda ordem & $419,96(131)$ & 0,93 & 0,94 & 0,07 \\
\hline
\end{tabular}

Nota: $\chi^{2}$ : Qui-quadrado; gl: Grau de liberdade; TLI: Tucker-Lewis Index; CFI: Comparative Fit Index; RMSEA: Root Mean Square Error of Approximation.

Tabela 3

Médias, Desvios Padrões e Coeficientes de Correlação entre as Escalas do Estudo

\begin{tabular}{|c|c|c|c|c|c|c|c|c|c|c|c|}
\hline & 1 & 2 & 3 & 4 & 5 & 6 & 7 & 8 & 9 & 10 & 11 \\
\hline \multicolumn{12}{|l|}{1 - Coragem } \\
\hline 2 - Temperança & 0,93 & & & & & & & & & & \\
\hline 3 - Justiça & 0,90 & 0,89 & & & & & & & & & \\
\hline 4 - Prudência & 0,91 & 0,85 & 0,99 & & & & & & & & \\
\hline 5 - Humanidade & 0,85 & 0,86 & 0,99 & 0,94 & & & & & & & \\
\hline 6 - Autoconsciência & 0,81 & 0,83 & 0,93 & 0,91 & 0,89 & & & & & & \\
\hline 7 - Transparência & 0,84 & 0,80 & 0,84 & 0,80 & 0,84 & 0,90 & & & & & \\
\hline 8 - Moral e ética & 0,83 & 0,82 & 0,92 & 0,90 & 0,88 & 0,94 & 0,85 & & & & \\
\hline 9 - Processamento balanceado & 0,82 & 0,80 & 0,87 & 0,88 & 0,84 & 0,91 & 0,83 & 0,92 & & & \\
\hline 10 - Satisfação no trabalho & 0,54 & 0,54 & 0,59 & 0,55 & 0,55 & 0,58 & 0,60 & 0,50 & 0,54 & & \\
\hline 11 - Afetos negativos & $-0,13$ & $-0,18$ & $-0,24$ & $-0,18$ & $-0,20$ & $-0,22$ & $-0,15$ & $-0,23$ & $-0,17$ & $-0,25$ & \\
\hline 12 - Afetos positivos & 0,51 & 0,47 & 0,64 & 0,62 & 0,59 & 0,60 & 0,61 & 0,68 & 0,59 & 0,75 & $-0,41$ \\
\hline
\end{tabular}

6 Nota: Todos os valores foram significativos $(p<0,01)$. 
Autêntica, com os resultados dessas correlações variando de $r=0,80 ; p<0,01$ a $r=0,93 ; p<0,01$, o que deu suporte à Hipótese 1 do estudo. Todos os fatores da Escala de Liderança Virtuosa também apresentaram correlações positivas altas com a Escala de Satisfação no Trabalho $(r=0,54 ; p<0,01$ a $r=0,59 ; p<0,01)$, além de que quatro desses fatores obtiveram correlações positivas altas com a subescala de afetos positivos, o que também deu suporte às Hipóteses 2 e 3 da investigação, respectivamente. Contudo, os fatores da escala de Liderança Virtuosa apresentaram uma correlação negativa, porém baixa, com a subescala de afetos negativos $(r=-0,13 ; p<0,01$ a $r=-0,24 ; p<0,01)$, o que impediu a sustentação da Hipótese 4.

\section{Discussão}

O presente trabalho teve como objetivo investigar as evidências de validade de estrutura interna, de relação com variáveis externas e de consistência interna da Escala de Liderança Virtuosa em uma amostra brasileira. Após a análise fatorial confirmatória, observou-se que o modelo de melhor ajuste foi o de cinco fatores de primeira ordem, o qual reproduziu integralmente a estrutura original do instrumento. Tais resultados confirmam, portanto, o modelo de Wang e Hacket (2016) em uma amostra brasileira.

Os índices de consistência interna dos fatores da escala também foram satisfatórios e assemelharam-se aos anteriormente verificados por Wang e Hacket (2016). Nesse sentido, a escala original apresentou índices iguais a 0,90 (coragem), 0,84 (temperança), 0,86 (justiça), 0,88 (prudência) e 0,87 (humanidade), enquanto, no presente estudo, esses índices foram iguais a 0,81 (coragem), 0,75 (temperança), 0,82 (justiça), 0,83 (prudência) e 0,88 (humanidade), ou seja, levemente inferiores aos obtidos pelos autores da escala.

No que diz respeito às evidências de validade da escala pela correlação com variáveis externas, foi possível verificar que todos os fatores da Escala de Liderança Virtuosa apresentaram uma correlação positiva alta com todos os fatores da Escala de Liderança Autêntica, o que deu suporte total à Hipótese 1. Tal correlação ratifica as semelhanças entre o estilo de liderança virtuosa e o de liderança autêntica, visto que ambos os líderes acabam por influenciar os liderados a partir de atitudes e comportamentos que possuem preceitos éticos e morais (Peterson, Walumbwa, Avolio, \& Hannah, 2012; Wang \& Hacket, 2016). Nesse sentido, a liderança autêntica possui os fatores de autoconsciência e transparência que mantêm estreita relação conceitual com os fatores de prudência e coragem da liderança virtuosa. Outrossim, os fatores de moral e ética guardam semelhanças com as dimensões de justiça e humanidade da liderança virtuosa, o mesmo ocorrendo entre o processamento balanceado (liderança autêntica) e a temperança (liderança virtuosa) (Hacket \& Wang, 2012; Peterson et al., 2012).

Os fatores da liderança virtuosa também apresentaram uma correlação positiva alta com a Escala de Satisfação no Trabalho e com os afetos positivos, o que forneceu suporte empírico às Hipóteses 2 e 3, respectivamente. Tais resultados convergem, portanto, com o argumento de que os líderes constituem modelos para seus subordinados, em razão do poder de referência a eles associado (Kilburg, 2012). Desse modo, eles acabam por influenciar positivamente esses indivíduos, ao motivá-los a emitir atitudes e comportamentos desejáveis ao bom convívio laboral (Atkinson \& Butler, 2012).

De acordo com Hacket e Wang (2012), a liderança virtuosa tende a gerar emoções e humores positivos, como prazer, entusiasmo e tranquilidade, e a diminuir as emoções e humores negativos, como ansiedade, raiva e depressão. Seria assim de se esperar uma correlação alta entre a liderança virtuosa e os afetos negativos. Contrariamente, porém, ao esperado, constatou-se uma correlação negativa baixa com os afetos negativos, o que impediu a sustentação da Hipótese 4. Uma possível explicação para tal resultado pode ser a de que os afetos positivos e negativos não constituem polos opostos de uma mesma dimensão, caracterizando-se, ao contrário, como dois sistemas distintos, com os afetos positivos associando-se a um sistema de abordagem, e os afetos negativos, a um sistema de evitação (Watson, Wiese, Vaidya, \& Tellegen, 
1999). Consequentemente, uma mesma característica do ambiente laboral não precisa necessariamente apresentar relações opostas com essas duas modalidades de afeto, ou seja, é plausível o fato de a liderança virtuosa ter mantido relações distintas com esses dois sistemas, ao apresentar alta correlação com os afetos positivos e baixa correlação com os afetos negativos. Em outras palavras, pode ser que a experiência com líderes virtuosos, que constitui um aspecto positivo do ambiente laboral, suscite tão somente uma abordagem de aproximação.

Outra provável explicação para tal resultado é a de que os afetos podem ser descritos não apenas por uma dimensão de valência (caracterizada pelos afetos positivos e negativos, como foi o caso da presente pesquisa), mas por duas dimensões bipolares, definidas pela valência (muito ou nenhum prazer) e pela ativação (alta ou baixa) (Warr, 1990). Da combinação dessas duas dimensões decorrem vários afetos, como, por exemplo, a ansiedade (nenhum prazer e alta ativação), o conforto (muito prazer e baixa ativação), a depressão (nenhum prazer e baixa ativação) e o entusiasmo (muito prazer e alta ativação) (Warr, 1990). É possível, assim, que a influência da liderança virtuosa sobre os afetos reflita processos mais específicos, que repercutem diferentemente em modalidades distintas de afetos, que variam em função de sua valência e ativação. De todo modo, tais explicações necessitam de futura verificação empírica.

Em síntese, os resultados ora obtidos indicam que a Escala de Liderança Virtuosa apresentou evidências de validade de estrutura interna, de relação com variáveis externas e de consistência interna, em uma amostra brasileira. Cumpre registrar, porém, que a amostra foi composta por participantes da região metropolitana e do interior do Estado do Rio de Janeiro e se caracterizou como uma amostra de conveniência, razão pela qual a generalização dos resultados ora obtidos deve ser feita com cautela. Considerando-se, outrossim, que a atual pesquisa incluiu categorias ocupacionais diversificadas, seria interessante que estudos futuros reunissem evidências adicionais de invariância da escala, mediante a comparação de sua estrutura interna em grupos ocupacionais específicos, tais como militares e gerentes de empresa, por exemplo.

Estudos futuros poderiam ainda aprofundar a rede nomológica do construto liderança virtuosa, especialmente no que diz respeito a seu impacto sobre o bem-estar no trabalho. A esse respeito, o fato de a liderança virtuosa ter apresentado apenas uma correlação fraca com os afetos negativos, contrariando o que era esperado, merece ser objeto de aprofundamento em investigações futuras, nas quais os afetos sejam mensurados de acordo com sua valência e ativação, e não apenas de acordo com sua valência. Tais pesquisas deveriam ser preferencialmente de natureza longitudinal, o que permitiria o aprofundamento da compreensão acerca dessas relações. De todo modo, as evidências iniciais de validade presentemente obtidas recomendam o uso futuro da Escala de Liderança Virtuosa em investigações brasileiras destinadas a avaliar o grau de liderança virtuosa e suas implicações para o bem-estar e qualidade de vida dos indivíduos no contexto laboral.

\section{Colaboradores}

M.C. FERREIRA foi responsável pela concepção e desenho do estudo, revisão e aprovação da versão final do manuscrito. M. PEREIRA participou da coleta, da análise e da interpretação dos dados do estudo, juntamente com M. GUIMARÃES que foi responsável pela elaboração do texto da versão final do manuscrito.

\section{Referências}

American Educational Research Association, American Psychological Association, \& National Council on Measurement in Education. (2014). Standards for educational and psychological testing. Washington, DC: Author. 
Atkinson, T. N., \& Butler, J. W. (2012). From regulation to virtue: A critique of ethical formalism in research organizations. Journal of Research Administration, 43(1), 17-32. http://dx.doi.org/10.1590/S0103-863X2012000300014

Bandura, A. (1977). Social learning theory. Englewood Cliffs: Prentice-Hall.

Barclay, L. A., Markel, K. S., \& Yugo, J. E. (2012). Virtue theory and organizations: Considering persons with disabilities. Journal of Managerial Psychology, 27(4), 330-346. http://dx.doi.org/10.1108/02683941211220153

Borsa, J. C., Damásio, B. F., \& Bandeira, D. R. (2012). Adaptação e validação de instrumentos psicológicos entre culturas: algumas considerações. Paidéia, 22(53), 423-432. http://dx. doi.org/10.1590/S0103863X2012000300014

Brown, T. A. (2015). Confirmatory factor analysis for applied research. New York: The Guilford Press.

Byrne, B. M. (2012). Structural equation modeling with Mplus: Basic concepts, applications, and programming. New York: Taylor \& Francis.

Cameron, K. (2011). Responsible leadership as virtuous leadership. Journal of Business Ethics, 98(1), 25-35. http://dx.doi. org/10.1007/s10551-011-1023-6

Cervo, C. S., Mónico, L. S. M., Santos, N. R., Hutz, C. S., \& Pais, C. (2016). Authentic Leadership Questionnaire: Invariance between samples of Brazilian and Portuguese employees. Psicologia: Reflexão e Crítica, 29(40), 1-11. http://dx.doi. org/10.1186/s41155-016-0046-4

Cohen, J. (1988). Statistical power analysis for the behavioral sciences (2nd ed.). Hillsdale: Lawrence Erlbaum Associates.

Crossan, M., Mazutis, D., \& Seijts, G. (2013). In search of virtue: The role of virtues, values, and character strengths in ethical decision making. Journal of Business Ethics, 113(4), 567-581. http://dx.doi.org/10.1007/s10551-013-1680-8

Daniels, K. (2000). Measures of five aspects of affective well-being at work. Human Relations, 53(2), 275-294. http:// dx.doi.org/10.1177/a010564

Ferreira, M. C., Silva, A. P. C., Fernandes, H. A., \& Almeida, S. P. (2008). Desenvolvimento e validação de uma escala de afetos no trabalho (ESAFE). Avaliação Psicológica, 7(2), 143-150. Recuperado em maio 1, 2017, de http://pepsic. bvsalud.org/scielo.php?script=sci_-arttext\&pid=S1677-04712008000200005\&lng=pt\&tIng=pt

Hackett, R. D., \& Wang, Q. (2012). Virtues and leadership: An integrating conceptual framework founded in Aristotelian and Confucian perspectives on virtues. Management Decision, 50(5), 868-899. http://dx.doi. org/10.1108/00251741211227564

Hart, D. (2001). Administration and the ethics of virtue: In all things, choose first for good character and then for technical expertise. In T. L. Cooper (Ed.), Handbook of administrative ethics (pp. 131-50). New York: Marcel Dekker.

Kilburg, R. R. (2012). Virtuous leaders: Strategy, character, and influence in the 21st century. Washington, DC: American Psychological Association.

Peterson, C., \& Seligman, M. E. P. (2004). Character strengths and virtues: A handbook and classification. Washington, DC: American Psychological Association.

Peterson, S. J., Walumbwa, F. O., Avolio B. J., \& Hannah, S. T. (2012). The relationship between authentic leadership and follower job performance: The mediating role of follower positivity in extreme contexts. The Leadership Quarterly, 23(3), 502-516. http://dx.doi.org/10.1016/j.leaqua.2011.12.004

Prottas, D. J. (2013). Relationships among employee perception of their manager's behavioral integrity, moral distress, and employee attitudes and well-being. Journal of Business Ethics, 113(1), 51-60. http://dx.doi.org/10.1007/s10551-0 $12-1280-z$

Riggio, R. E., Zhu, W., Reina, C., \& Maroosis, J. A. (2010). Virtue based measurement of ethical leadership: The leadership virtues questionnaire. Consulting Psychology Journal: Practice and Research, 62(4), 235-250. http://dx.doi.org/10.1037/ a0022286

Silva, A. P. C., \& Ferreira, M. C. (2009). Escala de satisfação geral no trabalho [Resumo]. Anais do IV Congresso Brasileiro de Avaliação Psicológica, p. 246. Campinas: IBAP. Recuperado em maio 1, 2017, de http://www.ibapnet.org.br/ congresso2009/ResumosPaineis.pdf

Sobral, F. J., \& Gimba, R. F. (2012). As prioridades axiológicas do líder autêntico: um estudo sobre valores e liderança. Revista de Administração Mackenzie, 13(3), 96-121. http://dx.doi.org/10.1590/\$1678-69712012000300006

Walumbwa, F., Avolio, B., Gardner, W., Wernsing, T., \& Peterson, S. (2008). Authentic leadership: Development and validation of a theory-based measure. Journal of Management, 34(1), 89-126. http://dx.doi.org/10.1177/0149206307308913

Wang, Q., \& Hackett, R. D. (2016). Conceptualization and measurement of virtuous leadership: Doing well by doing good. Journal of Business Ethics, 137(2), 321-345. http://dx.doi.org/10.1007/s10551-015-2560-1 
Warr, P. (1990). The measurement of well-being and other aspects of mental health. Journal of Occupational Psychology, 63(3), 193-210. http://dx.doi.org/10.1111/j.2044-8325.1990.tb00521.x

Watson, D., Wiese, D., Vaidya, J., \& Tellegen, A. (1999). The two general activation systems of affect: Structural findings, evolutionary considerations, and psychobiological evidence. Journal of Personality and Social Psychology, 76(5), 820-838. http://dx.doi.org/10.1037/0022-3514.76.5.820

Whetstone, J. T. (2001). How virtue fits within business ethics. Journal of Business Ethics, 33(2), 101-114. http://dx.doi. org/10.1023/A:1017554318867

Recebido: junho 30, 2017

Versão final: dezembro 6, 2017

Aprovado: fevereiro 9, 2018 